\title{
Simulation of radar backscatter and Doppler shifts of wave-current interaction in the presence of strong tidal current
}

\author{
M.W. Hansen ${ }^{a},{ }^{*}$, V. Kudryavtsev ${ }^{a, b, c}$, B. Chapron ${ }^{d}$, J.A. Johannessen ${ }^{a, e}$, F. Collard ${ }^{f}$, K.-F. Dagestad ${ }^{a}$, \\ A.A. Mouche ${ }^{f}$
}

\footnotetext{
${ }^{a}$ Nansen Environmental and Remote Sensing Center, Thormøhlensgate 47, NO-5006, Bergen, Norway

${ }^{\mathrm{b}}$ Nansen International Environmental and Remote Sensing Center, St. Petersburg, Russia

${ }^{c}$ Marine Hydrophysical Institute, Sebastopol, Ukraine

d Institut Français de Recherche pour l'Exploitation de la Mer, Plouzané, France

e Geophysical Institute, University of Bergen, Norway

${ }^{\mathrm{f}}$ Direction of Radar Applications, Collecte Localisation Satellites, Plouzané, France
}

*: Corresponding author : M. W. Hansen, Fax: +47 55205801 ; email address : morten.hansen@nersc.no

\begin{abstract}
:
A radar imaging model including a Doppler shift module is presented for quantitative studies of radar observations of wave-current interaction in a strong tidal current regime. The model partitions the Doppler shift into the relative contribution arising from the motion of the backscattering facets including Bragg waves, specular points, and breaking waves that are advected by and interact with the underlying surface current. Simulated and observed normalized radar cross sections and Doppler shifts for different environmental conditions and radar parameters are compared and discussed.
\end{abstract}

Keywords : SAR ; Tidal current ; Waves ; Normalized radar cross section ; Doppler velocity

\section{Introduction}

Airborne and spaceborne radar measurements at slanting incidence angles offer a method to map the ocean surface roughness linked to surface wind, waves and current, as well as to the presence of surface contaminants. Current shears affect the surface roughness leading to radar intensity-detectable patterns. For quantitative analysis of SAR measurements over the ocean, Kudryavtsev et al. (2005) and Johannessen et al. (2005) proposed a practical RIM of surface current features based on the NRCS model by Kudryavtsev et al. (2003a) 
Statistical properties of the sea surface result from a solution of the energy balance equation (e.g. Hughes (1978); Thompson (1988); Lyzenga and Bennett (1988)) where wind forcing, viscous and wave breaking dissipation, wave-wave interactions, and generation of shorter waves by breaking waves of longer scales are accounted for. The latter mechanism is described by Kudryavtsev and Johannessen (2004), and although it does not significantly alter the background spectrum, it plays a crucial role in the context of wave modulations by surface current (Kudryavtsev et al., 2005). The RIM thus consists of a particular decomposition of the sea surface into a regular wavy surface and a number of breaking zones. Radar scattering from the regular surface is described within the frame of the composite model combining specular reflection and resonant (Bragg) scattering waves with local tilting effects due to longer underlying waves (e.g. Plant (1986); Donelan and Pierson (1987); Romeiser et al. (1994); Romeiser and Alpers (1997)). The contribution from breaking waves can be described as specular reflections from very rough wave breaking patterns and is taken proportional to the fraction of the sea surface covered by breaking zones based on wave breaking statistics proposed by Phillips (1985).

Using Envisat Advanced SAR (ASAR) observations, Chapron et al. (2005) demonstrated the capability to use the Doppler centroid information embedded in the radar signal to map surface velocity, including wind-generated waves and current, from SAR images. The difference between a predicted Doppler shift based on precise knowledge of the satellite orbit and attitude, and the Doppler centroid frequency estimate in this case represents the geophysical Doppler shift experienced from the moving ocean surface. This geophysical Doppler shift in turn reflects the line-of-sight velocity of the scatterers, weighted by their contribution to the backscattered power (Romeiser and Thompson, 2000). The retrieval and subsequent error correction of the geophysical Doppler shift from the ASAR Wide Swath Medium resolution image (WSM) product is presented in Hansen et al. (2011a) where the accuracy of the geophysical Doppler shift is found to be about $5 \mathrm{~Hz}$. This corresponds to a horizontal surface velocity of $20 \mathrm{~cm} / \mathrm{s}$ at an incidence angle of $40^{\circ}$, and $40 \mathrm{~cm} / \mathrm{s}$ at an incidence angle of $20^{\circ}$. As such, the accuracy is still an issue in single scenes, although 
temporal averaging has been shown to capture the mean circulation in e.g. the Agulhas region (Rouault et al., 2010) and in the Norwegian Sea (Hansen et al., 2011b). The range Doppler velocity is not a direct surface current measurement, but the use of Doppler shift observations can help to provide valuable insights into the mesoscale dynamics to more quantitatively interpret high resolution radar roughness changes. The RIM model extended with a Doppler shift module was first presented by Johannessen et al. (2008) and follows the concept in RIM by treating the Doppler shift as a result of the partial contributions from the regular surface and breaking waves.

The objective of this paper is to further assess and demonstrate the combined approach to SAR image interpretation based on the use of both Doppler shift and RIM analysis. In Section 2, the Doppler shift equations and RIM are consistently combined into the Doppler Radar Imaging Model (DopRIM) as done in Johannessen et al. (2008), however, with a more detailed description of the contributions from the different scattering mechanisms. We do not consider SAR imaging artifacts such as e.g. velocity bunching. Model calculations providing total and partial contributions to the range Doppler velocity from each type of the scattering mechanisms for varying incidence angle and wind speed are presented in Section 3.1, including a comparison to the observed range Doppler velocity signal from ASAR WSM acquisitions over the Norwegian Sea. In Section 3.2, DopRIM calculations for a situation of strong tidal current in the Iroise Sea outside Brest, France, are compared to the NRCS and range Doppler velocity from an ASAR Single Look Complex (SLC) acquisition on 5 October 2005. Section 4 provides the summary and conclusion.

\section{The DopRIM Approach}

The Doppler shift of the radar backscatter from a moving target is given by $f_{D}=-k_{R} v / \pi$, where $k_{R}$ is the radar wavenumber, and $v$ is the line-of-sight velocity of the target (defined positive if directed away from the radar). Following a two-scale decomposition, it is suggested that the sea surface consists of an ensemble of small-scale scattering facets (with local NRCS $\left.\sigma_{0}\right)$ which cover a large scale surface formed by superposition of longer surface waves. These scattering facets experience vertical and horizontal movements due to the longer surface 
waves, resulting in a spatially variable $\sigma_{0}$ over the large-scale surface. In this case, the average Doppler shift reads (Romeiser and Thompson, 2000; Chapron et al., 2005):

$$
\frac{\pi f_{D}}{k_{R}}=-\frac{\overline{(u \sin \theta-w \cos \theta) \sigma_{0}(\theta+\Delta \theta)}}{\overline{\sigma_{0}(\theta+\Delta \theta)}} .
$$

Here, $u$ and $w$ are the horizontal and vertical velocities of the scattering facets in the radar incidence plane, and $\Delta \theta$ is the local modification of the incidence angle $\theta$ due to waves. The geometry in (1) is illustrated in Fig. 6 of Chapron et al. (2005). Following Johannessen et al. (2008), each parameter on the right side of (1) can be split as $y=\bar{y}+\tilde{y}$, where bar and tilde denote spatial mean and wave induced modulations. The latter is of order $\epsilon$, where $\epsilon$ is the steepness of the modulating longer waves. To the second order of $\epsilon$, (1) gives the following expression for the mean horizontal (ground) range Doppler velocity, $V_{D}$ :

$$
V_{D}=-\frac{\pi f_{D}}{k_{R} \sin \theta}=\bar{c}_{f}+u_{s}-\frac{1}{\tan \theta} \cdot \frac{\overline{\tilde{w} \tilde{\sigma}_{0}}}{\overline{\sigma_{0}}}+\frac{\overline{\tilde{u} \tilde{\sigma}_{0}}}{\overline{\sigma_{0}}},
$$

where $\bar{c}_{f}$ is the mean velocity of the scattering facets relative to the surface current, $u_{s}$ is the surface current including wind drift, and $\tilde{u}$ and $\tilde{w}$ are components of the orbital velocities of surface waves carrying the facets in the radar incidence plane. The last two terms on the right hand side of (2) describe the net contribution from the correlation of local NRCS variations with wave orbital motions. Following a general approach, RIM explains the local NRCS variations by changes of the local surface tilt and hydrodynamic modulation of the scattering facets, expressed as

$$
\tilde{\sigma}_{0}=\Delta \theta \frac{\partial \sigma_{0}}{\partial \theta}+\tilde{\sigma}_{0}^{h},
$$

where $\Delta \theta=-\left(\zeta_{1} \cos \phi_{R}+\zeta_{2} \sin \phi_{R}\right), \phi_{R}$ is the radar look direction, and $\zeta_{1}=\partial \zeta / \partial x_{1}$ and $\zeta_{2}=\partial \zeta / \partial x_{2}$ are components of the sea surface slope in an arbitrary coordinate system $\left(x_{1}, x_{2}\right)$. Note that we have ignored the effects of surface tilt out of the incidence plane in (3) which is of order $O\left(\epsilon^{2}\right)$, i.e. much less than the remaining terms which are of order $O(\epsilon)$. Invoking $\zeta=A e^{i \Phi}$ as the vertical displacement of the surface by harmonic modulating waves ( $\Phi=K_{j} x_{j}-\Omega t, K_{j}, \Omega$ and $A$ are phase function, wavenumber, frequency and amplitude correspondingly), the amplitude of the wave quantities in (2) and (3) can be written as: 
$\hat{w}=-i \epsilon C, \hat{u}_{j}=\kappa_{j} \epsilon C, \hat{\zeta}_{j}=\kappa_{j} \epsilon$, and $\hat{\sigma}_{0}^{h}=\bar{\sigma}_{0} \epsilon M_{f}^{h}$ for $j=\{1,2\}$, where $C=\Omega / K$ is phase velocity, $\epsilon=A K, \kappa_{j}=K_{j} / K$ is the unit wavenumber vector of the modulating wave, and $M_{f}^{h}$ is the hydrodynamic Modulation Transfer Function (MTF) for the facets (see e.g. Kudryavtsev et al. (2003b)). In general, the hydrodynamic MTF is a complex number, $M_{f}^{h}=M_{1 f}^{h}+i M_{2 f}^{h}$, where the real part, $M_{1 f}^{h}$, describes correlation of a scattering facet's modulations with the surface elevation, and the imaginary part, $M_{2 f}^{h}$, describes correlation with the surface slope.

If the scattering facets travel along a large-scale surface composed of a wide spectrum of long waves with $K<K_{L}$, where $K_{L}$ is the spectral cutoff linked to the scale of the facets, equation (2) can be written as

$$
V_{D}=u_{s}+\bar{c}_{f}+c_{f}^{T H} s_{L}^{2},
$$

where $s_{L}^{2}=\int_{K<K_{L}} K^{-2} B(\mathbf{K}) \mathrm{d} \mathbf{K}$ is the Mean Square Slope (MSS) of the large scale surface and $c_{f}^{T H}$ is the contribution of long waves through tilt and hydrodynamic modulation of the facets:

$$
c_{f}^{T H}=\int_{K<K_{L}}\left[\left(-M_{f}^{t} \cot \theta+M_{1 f}^{h}\right) \cos \left(\phi_{R}-\phi_{K}\right)+M_{2 f}^{h} \cot \theta\right] C K^{-2} B(\mathbf{K}) \mathrm{d} \mathbf{K} / s_{L}^{2},
$$

where $M_{f}^{t}=\partial\left(\ln \sigma_{0}\right) / \partial \theta$ is the tilt MTF, $B(\mathbf{K})$ is the $2 \mathrm{D}$ saturation spectrum of large-scale waves, and $\phi_{K}$ is the direction of $\mathbf{K}$. As follows from (5), the two first terms (tilt and real part of the hydrodynamic MTF) provide changes of sign in $c_{f}^{T H}$ when the radar look direction varies from down- to upwind. On the other hand, the effect of facet-slope correlation (third term in (5)) does not depend on radar look direction, and should provide down- and upwind asymmetry in the range Doppler velocity.

If Bragg scattering is the dominant scattering mechanism, then (4) with (5) corresponds to the model developed by Romeiser and Thompson (2000). For long quasi-monochromatic waves that travel along the radar look direction, (4) and (5) combine to

$$
V_{D}=u_{s}+\bar{c}_{f}+\frac{\epsilon^{2} c}{2}\left[\left(-M_{f}^{t}+M_{2 f}^{h}\right) \cot \theta+M_{1 f}^{h}\right],
$$

which also corresponds to equation (B16) suggested by Chapron et al. (2005). 
Yet, to be fully consistent with previous efforts (Kudryavtsev et al., 2003a, 2005) the NRCS of the sea surface, $\sigma_{0}^{p}$, must also incorporate facets corresponding to wave breaking zones, such as the proposed decomposition:

$$
\sigma_{0}^{p}=\sigma_{0 r}^{p}(1-q)+\sigma_{0 b} q,
$$

where $\sigma_{0 r}^{p}$ corresponds to the facets formed by the "regular" surface (at $p=$ Vertical transmitVertical receive (VV) or Horizontal transmit-Horizontal receive (HH) polarization), and $\sigma_{0 b}$ corresponds to very rough facets such as wave breaking zones covering the fraction $q$ of the sea surface. Accordingly, $\sigma_{0 r}^{p}$ is described within the frame of the composite model combining 2-scale Bragg scattering and specular reflections: $\sigma_{0 r}^{p}=\sigma_{s p}+\sigma_{b r}^{p}$. In this model, the radar returns from breaking waves are not polarized, as a Kirchhoff-like term, and can also be simply approximated as specular reflections. In consequence, we are in the following dealing with three types of scattering facets (Bragg waves, specular points and breakers), and their contribution to the Doppler velocity is considered below.

\subsection{Some background properties of RIM}

Each of the scattering mechanisms in (7) depends on the radar parameters and the wind speed, and their partial contributions to $\sigma_{0}^{p}$ defined as: $P_{b r}^{p}=(1-q) \sigma_{b r}^{p} / \sigma_{0}^{p}, P_{s p}^{p}=$ $(1-q) \sigma_{s p} / \sigma_{0}^{p}$, and $P_{w b}^{p}=q \sigma_{w b} / \sigma_{0}^{p}$ for Bragg, specular and wave breaking, respectively. Example calculations of these quantities are shown in Fig. 1 for wind speeds of 5, 10, and $15 \mathrm{~m} / \mathrm{s}$ in $\mathrm{VV}$ and $\mathrm{HH}$ polarization. As expected, pure specular reflection dominates the radar return at low incidence angle $\left(<20^{\circ}\right)$ for both polarizations, while the relative role of non-Bragg scattering (specular reflection from the regular surface and very rough facets) is stronger in $\mathrm{HH}$ than in $\mathrm{VV}$ at moderate incidence angle $\left(>20^{\circ}\right)$.

The polarization ratio is an important parameter indicating the role of non-Bragg scattering in the sea surface NRCS. Fig. 1(c), (f) and (i) shows the model C-band polarization ratio for the sea surface at 5,10 and $15 \mathrm{~m} / \mathrm{s}$ wind for two types of scattering models: the composite model (specular and 2-scale Bragg), as well as the full RIM including wave breaking statistics. The full model predictions are very similar to the experimental data, also as 


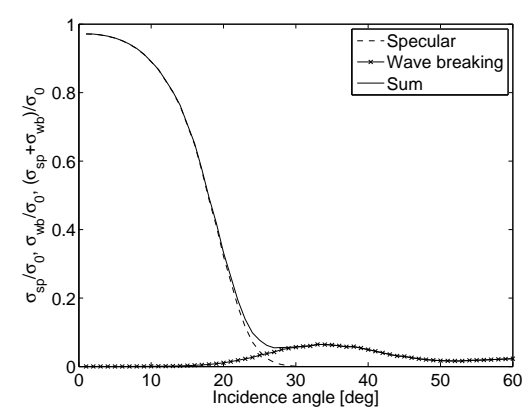

(a)

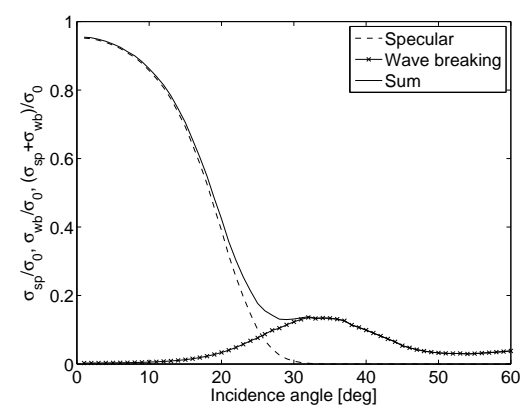

(d)

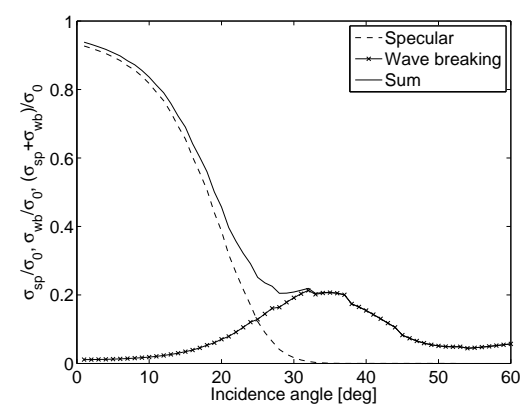

(g)

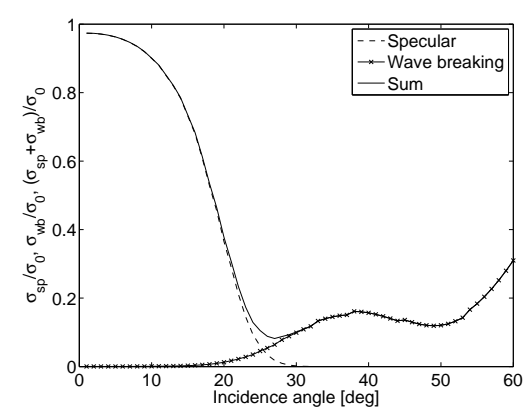

(b)

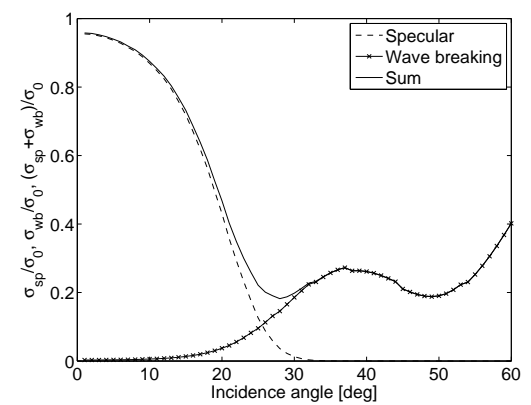

(e)

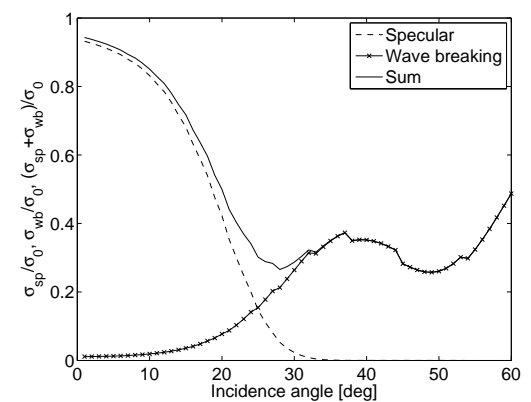

(h)

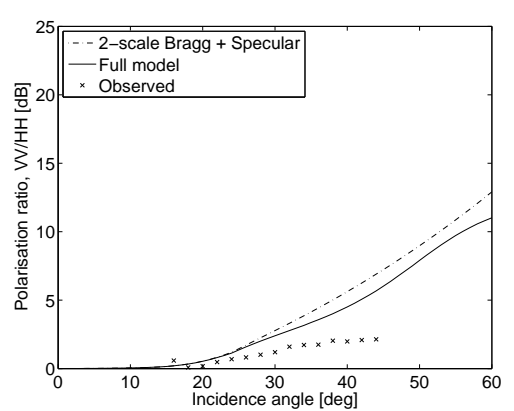

(c)

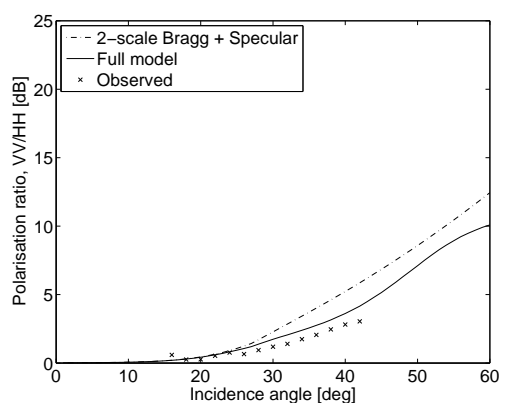

(f)

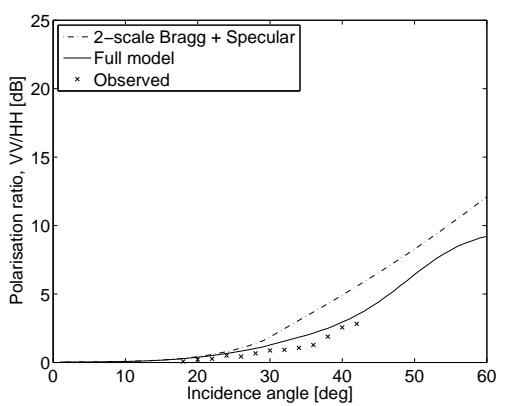

(i)

Figure 1: Partial contribution to the total NRCS of specular and wave breaking NRCS, and their sum, at wind speed of $5 \mathrm{~m} / \mathrm{s}$ (top row) $10 \mathrm{~m} / \mathrm{s}$ (center row) $15 \mathrm{~m} / \mathrm{s}$ (bottom row) in upwind configuration for VV (left column) and HH (center column) polarizations. Areas above the solid lines correspond to the partial contribution of Bragg scattering. The C-band polarization ratio for the sum of two-scale Bragg and specular reflection, for the full model, and from ASAR WSM observations over the Norwegian Sea (note that the average signal is here assumed to be wind dominated), is shown in the right column. 
reported e.g. by Mouche et al. (2006), except for some overestimation at $5 \mathrm{~m} / \mathrm{s}$ wind speed. A significant deviation of the composite scattering model prediction from the observations (similar to the full model) shows that the wave-breaking contribution plays an important role, and must be accounted for in the Doppler shift model. This correction could, however, in principle be more directly included using a more advanced scattering model (e.g. Mouche et al. (2007a), Mouche et al. (2007b)).

\subsection{Doppler shift estimate}

The simplified RIM NRCS as given by (7), will contribute to Doppler shifts associated with Bragg waves $(f \rightarrow b r)$, specular points $(f \rightarrow s p)$, and breakers $(f \rightarrow w b)$. This approach leads to the total range Doppler velocity

$$
V_{D}=u_{s}+\sum P_{j}^{p}\left(\bar{c}_{j}+c_{j}^{T H} s_{L}^{2}\right),
$$

where $\bar{c}_{j}$ and $c_{j}^{T H}$ are obtained for each of the scattering mechanisms, and $s_{L}^{2}$ is the MSS of the large-scale surface which is also different for each of the scattering mechanisms. Equation (8) is the governing equation of DopRIM. The input statistics needed to calculate the range Doppler velocity with (8) (e.g. various statistical properties of wind waves and different characteristics of the radar backscatter) are essentially taken from the RIM, which was described to detail in Kudryavtsev et al. (2005). We suggest that the surface wave field is a mixed sea consisting of wind generated waves and swell. We also assume that swell and wind waves are well separated in $k$-space, i.e. the peak wavenumber of wind waves, $k_{p}$, is much larger than the swell wavenumber: $k_{p} \gg k_{s w}$. The phase velocity of the waves is given by the dispersion relation, i.e. $c(k)=\omega / k=\sqrt{g / k+\gamma k}$ where $\omega$ is the wave frequency, $g$ is the gravitational acceleration and $\gamma$ is the surface tension. This is used in the calculation of the different contributions to $V_{D}$, as further outlined below.

\subsubsection{2-scale Bragg}

The velocity of facets corresponding to the Bragg waves is equal to the phase velocity $\bar{c}_{b r}=\bar{c}\left(k_{b r}\right)$. The high-frequency cutoff, $K_{L}$, of the large-scale surface in (5) then corresponds 
to the dividing wavenumber, $k_{d}$, of the 2 -scale Bragg model $\left(K_{L}=k_{d}=d k_{R}\right.$, with $\left.d=1 / 4\right)$.

The tilt MTF for Bragg scattering in (5) corresponds to

$$
M_{b r}^{t}=\frac{\partial\left(\ln \sigma_{0 b r}\right)}{\partial \theta} .
$$

In the present study, the wave spectrum modulations (prescribing the hydrodynamic MTFs for all types of facets in (5)) will be described in a simplified form, making use of the relaxation time approximation (see e.g. Alpers and Hasselmann (1978); Phillips (1984)). This accounts for the interaction of short waves with the orbital velocities of longer waves only (see Kudryavtsev et al. (2003b) for a detailed discussion of the MTF problem). In this case, the hydrodynamic MTF reads

$$
M^{h}(\mathbf{k}, \mathbf{K})=-\left(\frac{1-i \tau}{1+\tau^{2}}\right) \frac{k_{1}}{N(\mathbf{k})} \frac{\partial N(\mathbf{k})}{\partial k_{1}}
$$

where the "gradient" of the wave action spectrum $N$ in (10) is

$$
\frac{k_{1}}{N(\mathbf{k})} \frac{\partial N(\mathbf{k})}{\partial k_{1}}=\cos ^{2}\left(\phi-\phi_{K}\right) \frac{\partial \ln N}{\partial \ln k}-\frac{1}{2} \sin \left(2\left(\phi-\phi_{K}\right)\right) \frac{\partial \ln N}{\partial \phi}
$$

where $k_{1}$ is the wavenumber component of the modulated waves, $\mathbf{k}$, along the direction of the modulating waves (with wavenumber $K$ ), $\phi$ and $\phi_{K}$ are, respectively, the directions of short modulated and longer modulating waves, and $\tau$ is the dimensionless relaxation parameter. The latter quantity is defined as $\tau=n \beta \omega / \Omega$, where $\beta=c_{\beta}\left(u_{*} / c\right)^{2}$ is the growth rate of wind-waves, $c_{\beta}$ is a constant related to the growth rate, $\Omega$ and $\omega$ are the frequencies of the modulating and the modulated waves, respectively, and $n$ is the exponent of the spectrum in the parametrization of non-linear energy losses (see Kudryavtsev et al. (2003a) for details). For practical applications, the "wavenumber exponent", $m_{k} \equiv \partial \ln N / \partial \ln k$, can be evaluated approximately as $m_{k} \approx-9 / 2$ (e.g. as for the spectrum suggested by Phillips (1980)). Thus for a "typical" angular distribution of the Bragg-wave spectrum (say $N \propto \cos \phi)$, the second term in (11) is, in order of magnitude, less than the first one. Moreover, the hydrodynamic MTF appears in (5) under the integral over the modulating waves. Since the angular distribution of the large-scale surface (the range of equilibrium gravity waves) is approximately isotropic, the integral of the "oscillating" second term over 
the direction of the modulating waves is assumed to be small relative to the integral of the first term. Thus, hereinafter, the second term on the right-hand-side (rhs) of (11) is ignored. For the Bragg-facets, the hydrodynamic MTF (10) is now reduced to

$$
M_{b r}^{h}=m_{k} \cos ^{2}\left(\phi_{R}-\phi_{K}\right)\left(\frac{1-i \tau_{b r}}{1+\tau_{b r}^{2}}\right)
$$

where $\phi_{R}$ is the radar look direction, and $\tau_{b r}^{2}$ is the relaxation parameter taken at the Bragg wavenumber. Thus, the effect of tilt and hydrodynamic modulations of Bragg waves on the range Doppler velocity, $V_{D}$, is described by a combination of (5), (9), and (12), with $k_{L}=d k_{R}(d=1 / 4)$.

\subsubsection{Specular Reflection}

At low incidence angle $\left(15^{\circ}<\theta<25^{\circ}\right)$, the specular reflections from slopes of largescale waves with $k<k_{d}$ are important. The scattering facet velocity, $\bar{c}_{s p}$, in this case corresponds to the mean line-of-sight velocity of all facets with slopes providing specular reflections ("mirror points"). An expression for the mean velocity of these facets can be found in Longuet-Higgins (1957). In an orthogonal coordinate system (i, n) fixed to a radar look direction ( $\mathrm{i}$ and $\mathrm{n}$ axes along the incidence plane and normal to the incidence plane, respectively), the mean velocity of the mirror points in the radar look direction reads

$$
\overline{c_{i}}=\left(\overline{\zeta_{n} \zeta_{t}} \cdot \overline{\zeta_{i} \zeta_{n}}-\overline{\zeta_{i} \zeta_{t}} \cdot \overline{\zeta_{n} \zeta_{n}}\right) / \Delta_{2}
$$

where $\zeta_{i}=\mathrm{d} \zeta / \mathrm{di}$ and $\zeta_{n}=\mathrm{d} \zeta / \mathrm{dn}$ are the sea surface slopes along and normal to the incidence plane, $\zeta_{t}=\mathrm{d} \zeta / \mathrm{dt}$ is the time derivate of the sea surface elevation (i.e. the vertical velocity of the sea surface), and $\Delta_{2}=\left(\overline{\zeta_{i} \zeta_{i}} \cdot \overline{\zeta_{n} \zeta_{n}}-{\overline{\zeta_{i} \zeta_{n}}}^{2}\right)$ is the determinant of the covariance matrix of the sea surface slopes. It is more convenient to rewrite (13) in terms of up- and cross-wind surface slopes (i.e. $\zeta_{1}$ and $\zeta_{2}$, respectively). Given that $\zeta_{i}=\zeta_{1} \cos \phi_{R}+\zeta_{2} \sin \phi_{R}$, $\zeta_{n}=\zeta_{2} \cos \phi_{R}-\zeta_{1} \sin \phi_{R}$, and that $\overline{\zeta_{1} \zeta_{2}}=0$ (the latter for wind waves only), (13) is reduced to

$$
\overline{c_{i}}=-\frac{\overline{\zeta_{1} \zeta_{t}}}{\overline{\zeta_{1} \zeta_{1}}} \cos \phi_{R}-\frac{\overline{\zeta_{2} \zeta_{t}}}{\overline{\zeta_{2} \zeta_{2}}} \sin \phi_{R}
$$


or finally, in terms of the wind wave saturation spectrum,

$$
\bar{c}_{s p}=\frac{\cos \phi_{R}}{s_{L u p}^{2}} \int_{K<k_{d}} \cos \left(\phi_{K}\right) C K^{-2} B(\mathbf{K}) \mathrm{d} \mathbf{K}+\frac{\sin \phi_{R}}{s_{L c r}^{2}} \int_{K<k_{d}} \sin \left(\phi_{K}\right) C K^{-2} B(\mathbf{K}) \mathrm{d} \mathbf{K},
$$

where up- and cross-wind MSS of the "large-scale" waves $\left(s_{L u p}^{2}\right.$ and $s_{L c r}^{2}$, respectively) are defined as

$$
\left[s_{\text {Lup }}^{2}, s_{L c r}^{2}\right]=\int_{K<k_{d}}\left[\cos ^{2} \phi_{K}, \sin ^{2} \phi_{K}\right] K^{-2} B(\mathbf{K}) \mathrm{d} \mathbf{K} .
$$

Contrary to the 2-scale Bragg scattering model, the specular reflections model does not possess a spectral gap between short waves providing radar reflections, and longer wind waves which would tilt and modulate these waves. As follows from $(16), s_{L}^{2}=\int B(K) \mathrm{d} \ln K$. Thus, if the omni-directional spectrum $B(K)$ is approximately constant (this corresponds to wind seas), all the waves almost equivalently contribute to the MSS, and there is no reason to introduce the effect of facet modulations by the dominant wind waves.

On the other hand, the existence of a mixed sea (swell plus wind waves) is very plausible in the open ocean. In this case, the spectral gap between specular facets and modulating long waves (swell) is obvious. We should therefore include the effect of swell on the range Doppler velocities through tilt and hydrodynamic modulation of the specular facets - the term $c_{s p}^{T H}$ in (8). Thus, the large-scale waves in (5) now correspond to swell. The tilt MTF in (5) is then $M_{s p}^{t}=\partial\left(\ln \sigma_{s p}\right) / \partial \theta$, while $M_{s p}^{h}$ in (5) corresponds to the hydrodynamic modulation of the specular point density due to modulation of the MSS, $s_{L}^{2}=\int_{k_{p}}^{k_{d}} K^{-2} B(\mathbf{K}) \mathrm{d} \mathbf{K}$, of the wind waves (reminding that $k_{p}$ is the spectral peak wavenumber of the wind-generated waves). With the use of the well-known expression for $\sigma_{s p}$ (see e.g. equation (10) in Kudryavtsev et al. (2005)), the linear hydrodynamic MTF for $\sigma_{s p}$, due to modulations of the MSS, is expressed as

$$
M_{s p}^{h}=\left(\frac{\tan ^{2} \theta}{s_{L}^{2}}-1\right) \int_{k_{p}}^{k_{d}} M^{h}\left(\phi-\phi_{s w}\right) B(k, \phi) \mathrm{d}(\ln k) \mathrm{d} \phi / s_{L}^{2},
$$

where $\phi_{s w}$ is the swell direction, $M^{h}$ is given by (10) with (11) where (we remind) the second term on the rhs is omitted. 
The swell spectrum is normally very narrow, so its impact on $V_{D}$ through tilt and hydrodynamic modulation of specular points can be expressed as

$$
c_{s p}^{T H}=C_{s w}\left[\cos \left(\phi_{R}-\phi_{s w}\right)\left(-M_{1 s p}^{t} \cot \theta+M_{1 s p}^{h}\right)+M_{2 s p}^{h} \cot \theta\right]
$$

where $C_{s w}$ and $\phi_{s w}$ are the phase velocity and direction of the swell. The "long-wave" MSS (i.e. the swell MSS) is here defined as $s_{L}^{2}=A_{s w}^{2} K_{s w}^{2} / 2$, where $A_{s w}$ and $K_{s w}$ are swell amplitude and wavenumber.

\subsubsection{Wave Breaking}

The distribution of breakers over the wave scales can be described in terms of $\Lambda(\mathbf{c}) \mathrm{d} \mathbf{c}$, which defines the length of wave breaking fronts per unit area with velocities ranging from $\mathbf{c}$ to $\mathbf{c}+\mathrm{d} \mathbf{c}$ (Phillips, 1985). Assuming that the quantity $k^{-1} \Lambda(\mathbf{c}) \mathrm{d} \mathbf{c}$ is proportional to the fraction of the sea surface covered by these breakers, the mean breaker velocity weighted over all breakers (term $\bar{c}_{w b}$ in (8)) reads

$$
\bar{c}_{w b}=\int_{k<k_{w b}} \cos \left(\phi-\phi_{R}\right) c k^{-1} \Lambda(\mathbf{c}) \mathrm{d} \mathbf{c} / \int_{k<k_{w b}} k^{-1} \Lambda(\mathbf{c}) \mathrm{d} \mathbf{c},
$$

where $k_{w b}=k_{R} / 10$ is the wavenumber of the shortest breaking waves providing radar returns (Kudryavtsev et al., 2003a).

Longer waves also tilt the breakers and modulate their surface density. It is thus assumed that the wave breaking at wavenumber $k$ is tilted and modulated by longer waves with $K<d k$ (where $d=1 / 4$ as specified before). Following Phillips (1985), Kudryavtsev et al. (2003a) suggested that $\Lambda(\mathbf{c})$ is proportional to the saturation spectrum to the power $\left(n_{g}+1\right)$, with $n_{g}=5$ in RIM. Therefore, the MTF for the breaking front surface density modulations caused by longer waves with wavenumber $K$, reads

$$
\begin{aligned}
M_{w b}^{h}(\mathbf{K}) & =\left(n_{g}+1\right) \int_{K / d}^{k_{w b}} M^{h}(\mathbf{K}, \mathbf{k}) k^{-1} \Lambda(\mathbf{c}) \mathrm{d} \mathbf{c} \\
& =\left(n_{g}+1\right) \int_{K / d}^{k_{w b}} M^{h}(\mathbf{K}, \mathbf{k}) \beta B(\mathbf{k}) \mathrm{d}(\ln k) \mathrm{d} \phi
\end{aligned}
$$


with $M^{h}$ defined by (10) with 11) where (we remind) the second term on the rhs is omitted. In the second equality of (20), we have assumed that the velocity of the breaking crest of a wave at given wavenumber approximately obeys the linear dispersion relation, and that wave breaking provides most of the energy losses in wind waves. This is compensated by the energy input from the wind (Phillips, 1985; Kudryavtsev et al., 2003a). The integral, $\int \beta B(\mathbf{k}) \mathrm{d}(\ln k) \mathrm{d} \phi \propto k^{1+1 / n_{g}}$, converges rapidly at the upper limit of the integration. This means that the main contribution to any wave breaking quantity is coming from the shortest breaking waves, and there should be a spectral gap between the dominant breaking facets and modulating longer waves. Recognizing that $M_{w b}^{h}(\mathbf{K}) \propto 1-\left(K / d k_{w b}\right)^{1+1 / n_{g}}$, the MTF in (20) does not depend on the wavenumber of the modulating waves as long as $K$ is sufficiently small. A 2-scale model with an upper wavenumber limit, $k_{L}=k_{w b} / 10$, for longer waves which modulate the breaking facets, may therefore be introduced. This provides $70 \%$ of the "available" hydrodynamic modulations of the breaking facets.

In order to further simplify the problem we mention that, in the range of short breaking waves, the angular distribution of the wave spectrum is $\cos ^{2 / n_{g}}$, which is significantly broader than the angular distribution in $\beta\left(\propto \cos ^{2} \phi\right)$. This allows us to analytically evaluate integrals over $\phi$. Finally, the hydrodynamic MTF for breaking facets needed for (5) and (8) can, with the use of (20), be written approximately as

$$
\begin{aligned}
M_{w b}^{h}(\mathbf{K}) & =\left(n_{g}+1\right) \int_{K / d}^{k_{w b}} M^{h}(\mathbf{k}, \mathbf{K}) k^{-1} \beta B(\mathbf{k}) \mathrm{d} \mathbf{k} \\
& =-\frac{1}{4} m_{k}\left(n_{g}+1\right)\left(1+2 \cos ^{2} \phi_{K}\right) \frac{1-i \tau_{w b}}{1+\tau_{w b}^{2}},
\end{aligned}
$$

where $\phi$ is the direction of the modulating waves with wavenumber $K<k_{w b} / 10$, and $\tau_{w b}$ is the relaxation parameter estimated for breaking waves with $k=k_{w b}$. This equation predicts very strong modulation of the wave breaking with magnitude of $M_{w b}^{h} \approx 20$. This estimate is consistent with experimental findings reported by Dulov et al. (2002), as shown in Fig. 4 of Kudryavtsev et al. (2003b).

Tilt and hydrodynamic modulation, $c_{w b}^{T H}$, of the breaking waves to $V_{D}$ is, thus, given by (5) with the high-frequency cut-off of the modulating waves $k_{L}=k_{w b} / 10$, the hydrodynamic 
MTF described by (21), and the tilt MTF given through the NRCS of wave breaking as $M_{w b}^{t}=\partial\left(\ln \sigma_{0 b}\right) / \partial \theta$

\section{DopRIM Capabilities}

We present the influence of varying incidence angle and wind speed on the range Doppler velocity and the contributing scattering mechanisms in section 3.1. In section 3.2, we then present a case study to compare model simulations with Envisat ASAR observations for a situation of strong tidal current in the Iroise Sea outside Brest, France. In particular, we investigate modulations associated to the impact of wave breaking.

\subsection{Importance of incidence angle and wind speed}

The model calculations presented in the following are performed for pure developed wind seas, without swell, for a C-band radar. The total and partial contributions to the range Doppler velocity at $5 \mathrm{~m} / \mathrm{s}, 10 \mathrm{~m} / \mathrm{s}$, and $15 \mathrm{~m} / \mathrm{s}$ wind speed for each type of the scattering mechanisms are shown in Fig. 2. The velocity of the breaker-facets appears weakly dependent on incidence angle, with some excess at $\theta<45^{\circ}$ which results from tilting by larger scale waves. This vanishes at larger incidence angles. An "undulating" shape of the curves representing the partial contributions, $\frac{P_{j}^{p}\left(\bar{c}_{j}+c_{j}^{T H}\right)}{\sum P_{j}^{p}\left(\bar{c}_{j}+c_{j}^{T H}\right)}$, for each type of facets to the total range Doppler velocity at $5 \mathrm{~m} / \mathrm{s}, 10 \mathrm{~m} / \mathrm{s}$, and $15 \mathrm{~m} / \mathrm{s}$ wind speed is a consequence of the partial contribution of wave breaking fronts to the NRCS shown in Fig. 1 (which also demonstrates a similar undulation, but less pronounced). This is to some degree considered as an artifact resulting from slightly imperfect tuning of the wave breaking parameters, which was originally proposed by Kudryavtsev et al. (2003a) for a rather different purpose. The velocity of the mirror points dominates $V_{D}$ at low incidence angle. At moderate incidence angle, the effect of slightly rough facets play a dominant role in VV. For large incidence angles in HH polarization $\left(\theta>60^{\circ}\right.$ for $5 \mathrm{~m} / \mathrm{s}$ and $\theta>35^{\circ}$ for $10 \mathrm{~m} / \mathrm{s}$ or higher wind speed), the breakers dominate $V_{D}$. Their role in $\mathrm{VV}$ is less pronounced but approaches the contribution from slightly rough facets at larger incidence angle and higher wind speed. 


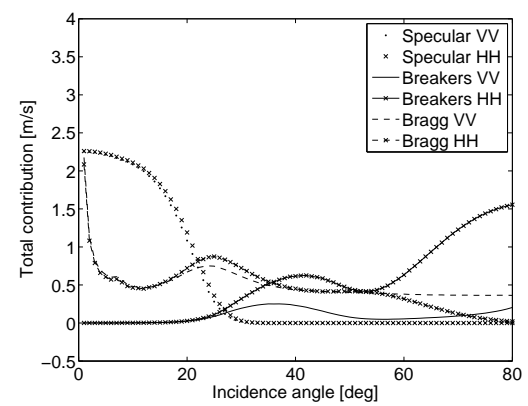

(a)

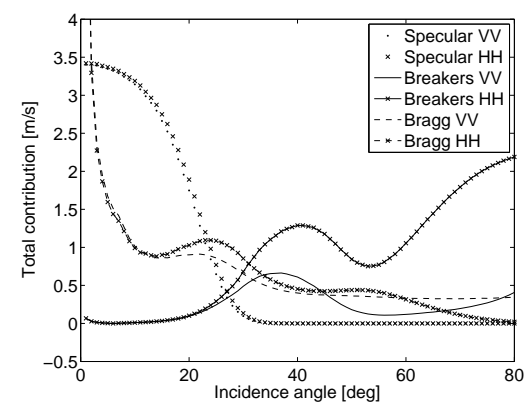

(d)

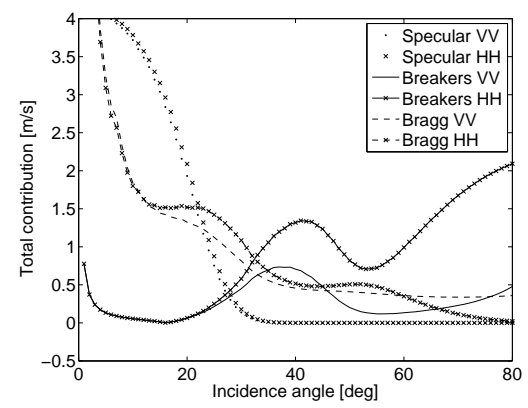

(g)

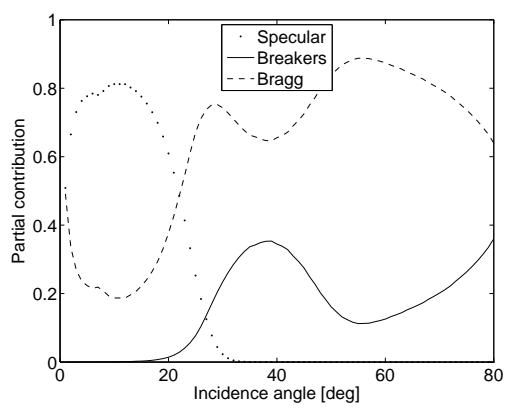

(b)

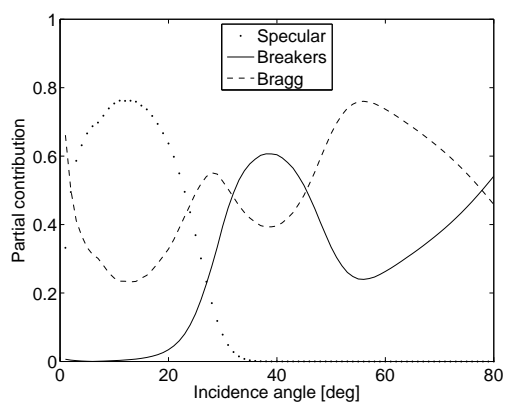

(e)

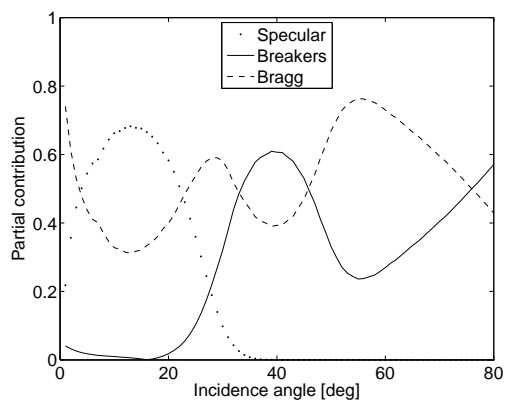

(h)

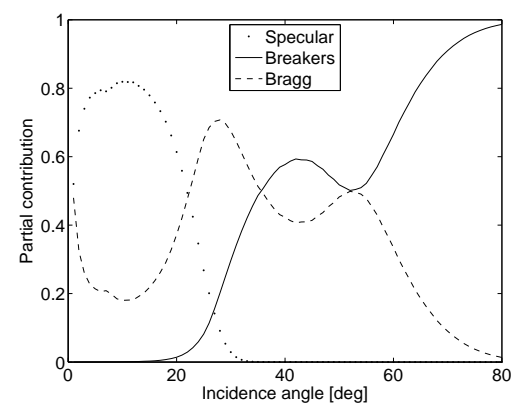

(c)

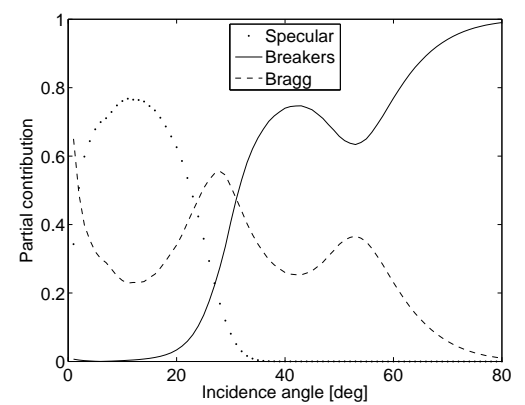

(f)

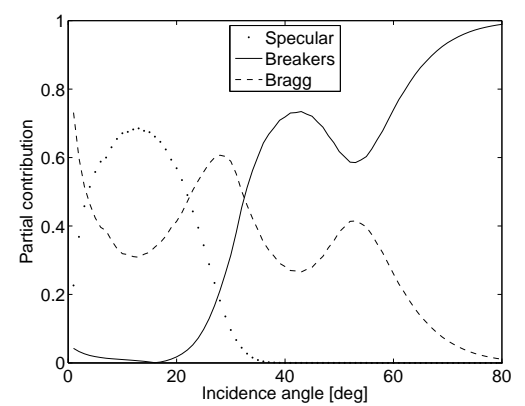

(i)

Figure 2: Total (left column) and partial (center and right column) contributions $P_{j}^{p}\left(\bar{c}_{j}+c_{j}^{T H} s_{L}^{2}\right)$ and $P_{j}^{p}\left(\bar{c}_{j}+c_{j}^{T H} s_{L}^{2}\right) /\left(V_{D}-u_{s}\right)$, respectively, for each type of facets at $5 \mathrm{~m} / \mathrm{s}$ (top row), $10 \mathrm{~m} / \mathrm{s}$ (center row), and $15 \mathrm{~m} / \mathrm{s}$ (bottom row) wind speed. The center/right column is for VV/HH polarization. All plots are for the downwind configuration. 
The dependence of the total range Doppler velocity, $V_{D}$, on incidence angle for $\mathrm{VV}$ and $\mathrm{HH}$ polarizations at wind speeds of $5 \mathrm{~m} / \mathrm{s}, 10 \mathrm{~m} / \mathrm{s}$, and $15 \mathrm{~m} / \mathrm{s}$ are shown in Fig. 3 for both up- and downwind configurations. At low incidence angles $\left(15<\theta<25^{\circ}\right)$, the range Doppler velocity is relatively large, with mean values reaching $37 \%(5 \mathrm{~m} / \mathrm{s}), 30 \%(10 \mathrm{~m} / \mathrm{s})$ and $26 \%(15 \mathrm{~m} / \mathrm{s})$ of the wind speed. This is much larger than expected from the phase speed of the Bragg waves (about $0.3 \mathrm{~m} / \mathrm{s}$ ) and the wind induced surface drift (about $3 \%$ of the wind speed), and it is thus evident that the contribution from other sources (i.e. the mean velocities of specular and breaking facets, and the correlation between the orbital motion of waves and the NRCS) must be accounted for. At larger incidence angles, there is a general decrease in $V_{D}$, except for $\mathrm{HH}$ polarization which reveals an increase in velocity at grazing angles. This results from the growing role of very rough patches and their modulation of the range Doppler velocity in HH. There is also a clear asymmetry between the range Doppler velocity in the up- and downwind configurations. This illustrates the effect of the facet-slope correlation (see (5)) which does not depend on the radar look direction.

As demonstrated in Johannessen et al. (2008), the present model compares well with Doppler shift observations from global Envisat ASAR WSM data in VV and HH polarization at incidence angles of $23^{\circ}$ and $33^{\circ}$. This is further confirmed by the comparison of observed and modeled range Doppler velocities in Fig. 3 for VV polarization. In $\mathrm{HH}$ polarization, however, there is some overestimation of $V_{D}$ for the upwind configuration at $10 \mathrm{~m} / \mathrm{s}$ and $15 \mathrm{~m} / \mathrm{s}$ wind speed. This could probably be improved by a better model fit, but until recently the amount of observed data in $\mathrm{HH}$ has been too low. Nevertheless, the non-Bragg mechanism is seemingly well captured by the proposed approach, and greatly simplifies a more advanced approach (e.g. Pedersen et al. (2004); Mouche et al. (2008)). The modeled Doppler shift, here, displays a functional relationship with wind speed in good agreement with the observations, particularly up to a wind speed of about $15 \mathrm{~m} / \mathrm{s}$. In the following section, we compare modeled and observed Doppler velocities as well as the corresponding NRCS for a specific case of wave-current interaction in the presence of strong tidal current in the Iroise Sea outside Brest, France. 


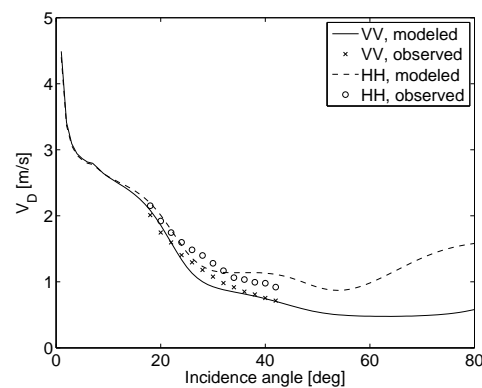

(a)

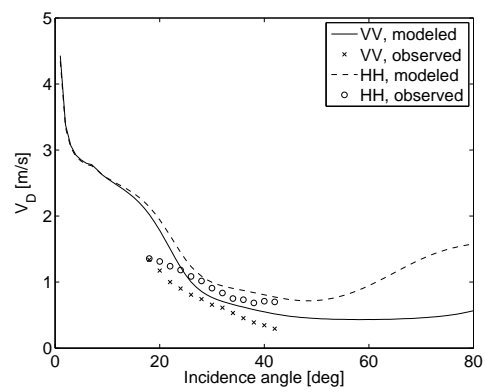

(d)

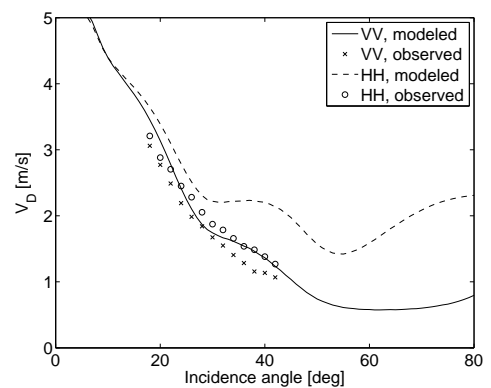

(b)

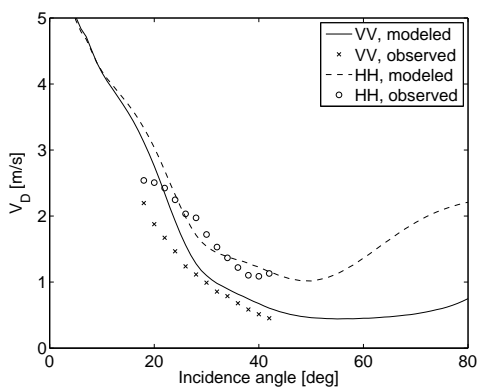

(e)

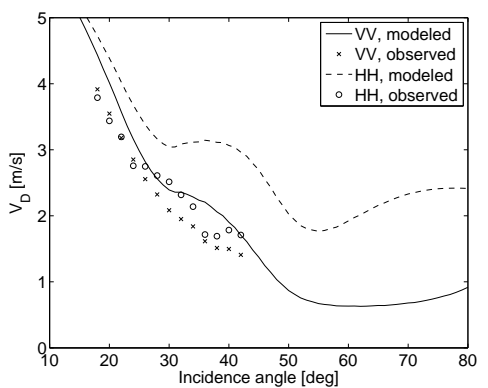

(c)

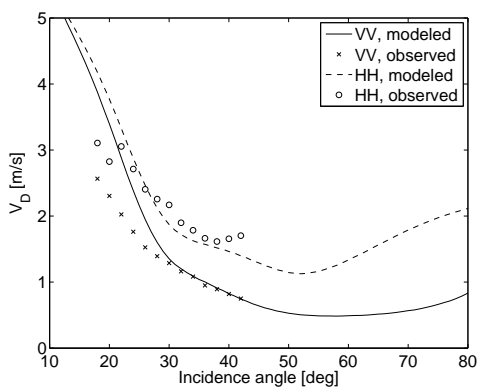

(f)

Figure 3: Range Doppler velocities for VV and HH polarization versus incidence angle at wind speed of $5 \mathrm{~m} / \mathrm{s}$ (left column), $10 \mathrm{~m} / \mathrm{s}$ (center column), and $15 \mathrm{~m} / \mathrm{s}$ (right column) in up- (top row) and downwind (bottom row) configuration. $3 \%$ wind drift is included in $V_{D}$. The observations represent the median range Doppler velocities at the given wind conditions retrieved from nearly 2200 ASAR WSM acquisitions over the Norwegian Sea from August 2007 to February 2011 (about 1200 in VV and 1000 in HH polarization, respectively). 


\subsection{NRCS and range Doppler velocity in the presence of strong tidal current}

Provided the sea surface state including near surface wind and current is known, DopRIM simulations can be assessed and compared to SAR NRCS and range Doppler velocity retrievals in order to improve the SAR image interpretation. Thanks to the availability of a 2-D numerical tide model (Le Nestour, 1993), the Iroise Sea (Brest coast, France) was chosen as a test area for the DopRIM simulations carried out in two steps: (i) Calculation of the components contributing to the NRCS and their modulations by the surface current with use of RIM (described to detail in Kudryavtsev et al. (2005)), and (ii) calculations of the range Doppler velocity field using (8) with the modeled NRCS field and the related statistical properties of its components (after RIM simulations). Notice that the facet velocities, $\bar{c}_{j}$, as well as the velocities $c_{j}^{T H}$ describing the impact of tilt and hydrodynamic modulations on $V_{D}$ via (8) are defined as weighted over the wave spectrum. Therefore they are weakly sensitive to the wave spectrum modulations due to wave-current interaction. The governing effect of wave-current interaction on $V_{D}$ appears via modulations of the MSS of the large-scale surface, $s_{L}^{2}$, and redistribution of the contribution from the different scattering mechanisms to the total NRCS, $P_{j}^{p}$. In particular, enhancement (suppression) of wave breaking in the current convergence (divergence) zones results in $V_{D}$ response via the partial contribution of radar backscatter from breaking waves to the total NRCS.

Because of limited coverage of the numerical tide model, the resolution of the range Doppler velocity from ASAR WSM acquisitions is too low to provide any reasonable comparison with the modeled NRCS and range Doppler velocity fields. However, by using the phase and amplitude information in ASAR SLC data, we have estimated the Doppler centroid frequency using Madsen's method (Madsen, 1989) and chosen a higher spatial resolution (600 $\mathrm{m}$ in range direction and $1600 \mathrm{~m}$ in azimuth direction) than given in the range Doppler velocity from the ASAR WSM products. The case we present here is one rare case where high resolution current information is available coincident with an ASAR SLC image.

The surface current field (input for DopRIM) obtained from the numerical tide model at the time of ASAR acquisition is shown in Fig. 4(a), and depicts large spatial variations 
with the current speed reaching up to $1.1 \mathrm{~m} / \mathrm{s}$ in the gap between the islands in northwest. The wind stress governing the short wind waves varies as the atmospheric boundary layer is adjusted to the sea surface temperature and current. A modified resistance law, incorporated in DopRim, relates surface friction velocity $\left(u_{*}\right)$ to the geostrophic wind velocity $(G)$ and the surface current $\left(u_{s}\right)$ :

$$
u_{*}^{2}=C_{d G}\left|\mathbf{G}-\mathbf{u}_{\mathbf{s}}\right|^{2},
$$

where $C_{d G}$ is the geostrophic drag coefficient depending on the atmospheric stratification parameter, $\mu$ (see Kudryavtsev et al. (2005)).The friction velocity was obtained from (22) for a geostrophic wind speed of $G=6.0 \mathrm{~m} / \mathrm{s}$ from northeast (calculated from a wind speed of $4.4 \mathrm{~m} / \mathrm{s}$ at $10 \mathrm{~m}$ height following Kudryavtsev et al. (2000)) under neutral stratification, and is shown in Fig. 4(b). As anticipated, the shape of the $u_{*}$ field is very similar to the pattern of the current field. In particular, the strong southwesterly tidal currents, exceeding $1.1 \mathrm{~m} / \mathrm{s}$ between the outer islands and to the north of the island, lead to significant drops in the friction velocity.

These sea surface current and wind stress fields are input to the DopRIM simulations. The simulated contrasts, defined as $\left(Y(x, y)-Y_{0}\right) / Y_{0}$ where $Y_{0}$ is the background signal induced by wind stress, for the Bragg wave spectrum $\left(Y=\sigma_{b r}\right)$ and the MSS of large scale waves with $k<d k_{R}\left(Y=s_{L}^{2}\right)$ are shown in Fig. 4(c) and 4(d). The Bragg waves feel the divergence of the current field, and also indirectly the surface current through the wind stress adjustment. The spatial variation of the Bragg roughness contrast is quite large near the outer islands (about a factor 3 or more, equivalent to $5 \mathrm{~dB}$ ). Since the wind stress variation in this area is about $20 \%\left(\left[u_{*, \max }-u_{*, \min }\right] / u_{*, \max }\right)$, we conclude that the impact of the wind stress adjustment on the Bragg waves is much weaker than the impact from the enhancement/suppression of wave breaking in the zones of surface current convergence/divergence. Indeed, the direct effect of current changes to short waves is negligible owing to the weak relaxation rate and, thus, the roughness modulation by intermediate wave breaking appears as the dominant source in the presence of a current (Johannessen et al., 2005).

The pattern of the MSS contrasts, on the other hand, differs significantly from the Bragg 


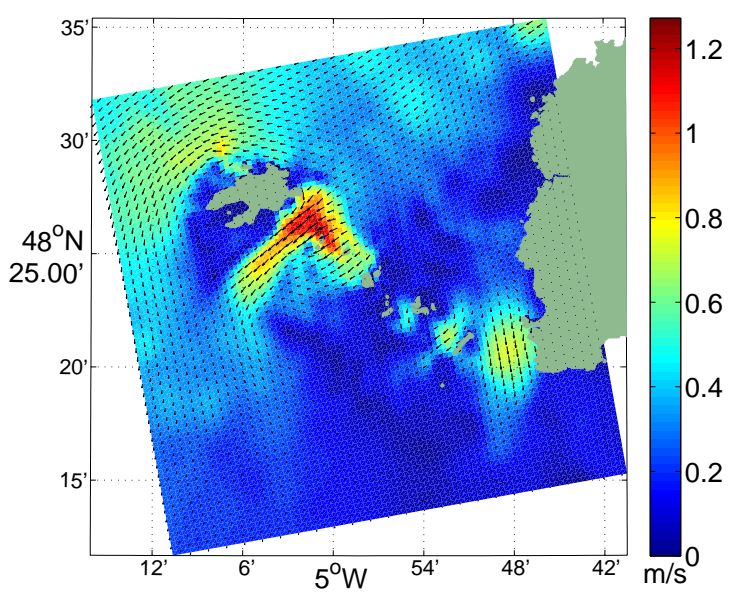

(a)

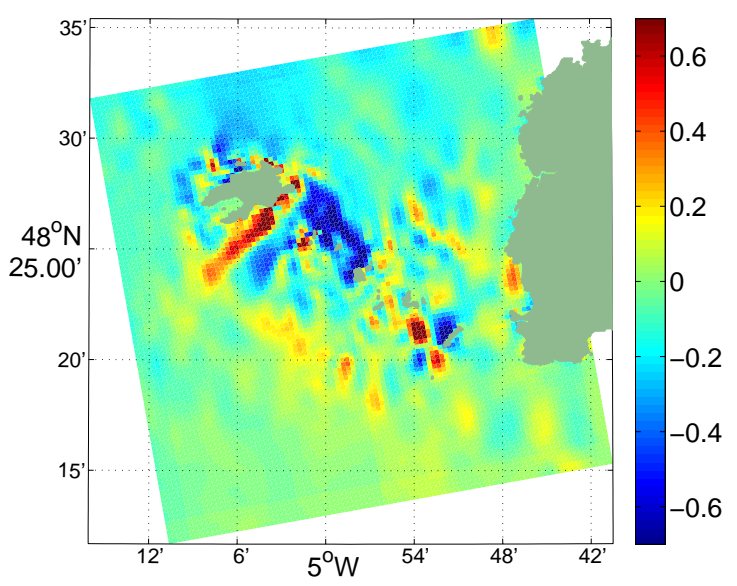

(c)

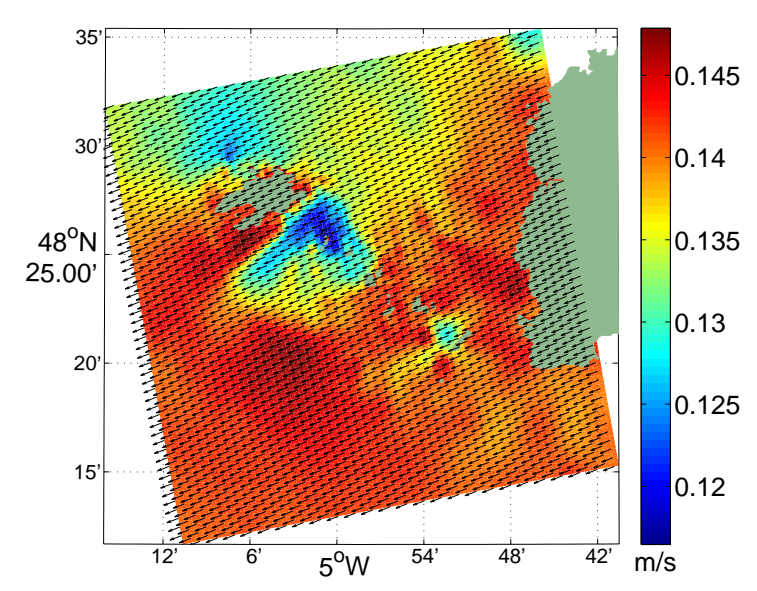

(b)

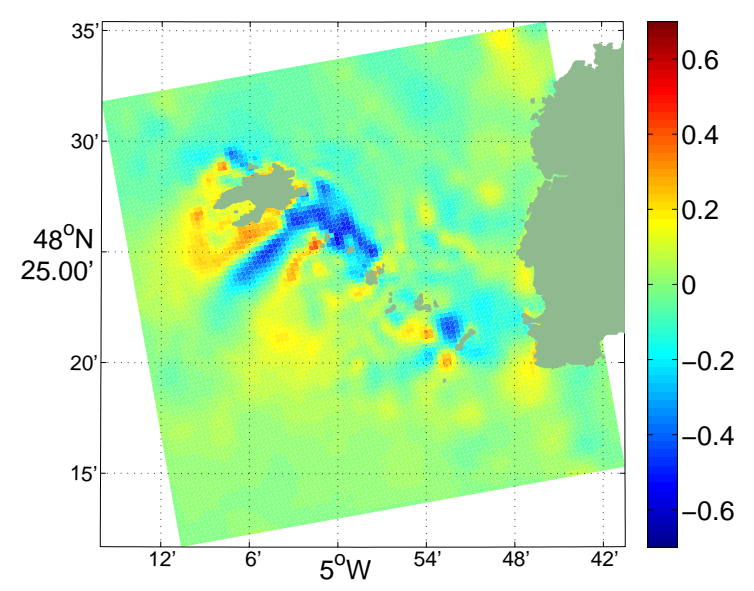

(d)

Figure 4: Model tidal current (a) and resulting friction velocity (b), roughness contrast induced by the Bragg wave spectrum (c), and MSS contrast of the large-scale waves (d) at 22:10 UTC on 5 October 2005. The mean wind speed at $10 \mathrm{~m}$ height was $4.4 \mathrm{~m} / \mathrm{s}$ from northeast. 


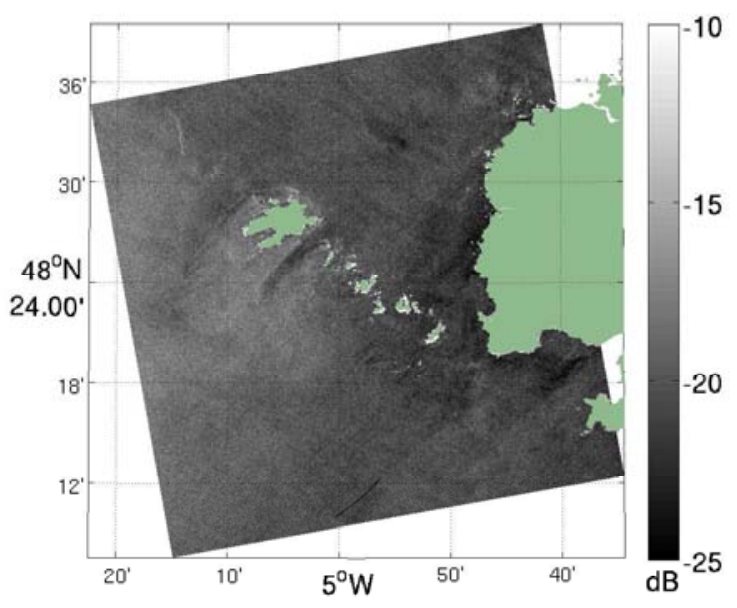

(a)

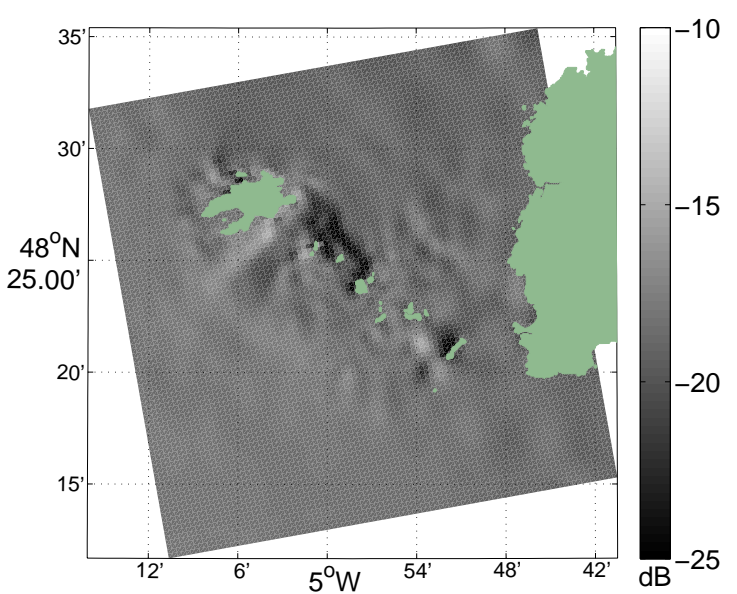

(b)

Figure 5: Observed (a) and simulated (b) NRCS on 5 October 2005 at 22:10 UTC. The ASAR data is in VV polarization and was obtained in ascending pass. This is the fifth subswath (IS5), and the image sizes are equal at about $38 \times 38 \mathrm{~km}$, which is a fragment of the full subswath image. The look direction is about $10^{\circ}$ with respect to the east, with incidence angles (for the subset) ranging from $36.5^{\circ}$ to $38.5^{\circ}$.

wave contrasts. Since the spatial scales of the relaxation of the long wind waves and the current deformation is of similar order, the MSS field predominantly possesses a contrast structure imposed by the large-scale patterns of the current field, such as the vorticity leading to the focusing of the wave trains downwind of the islands.

Finally, the simulated NRCS (Fig. 5(b)) reveals a structure resulting from the combined impact of Bragg waves, MSS, and wave breaking. Notice that for an incidence angle of about $37^{\circ}$ in this specific case, the unperturbed background radar scattering is mainly provided by Bragg scattering mechanism, while radar returns from breaking waves provide about $6 \%$ of the total NRCS at the given wind speed (see Fig. 1). In particular, there is evidence of a strong suppression between the islands and the enhancement downwind of the main island. Compared to the ASAR image (Fig. 5(a)), the mean level of the NRCS is similar $(-19 \mathrm{~dB})$, and the largest contrasts are depicted in the vicinity of the two outer islands in both images.

The range projected (horizontal) model current and the contribution of the surface roughness and its modulation to $V_{D}$ (see (8)) are depicted in Fig. 6(a) and 6(b), respectively. The simulated and the observed range Doppler velocity is shown in Fig. 6(c) and 6(d). Distinct 


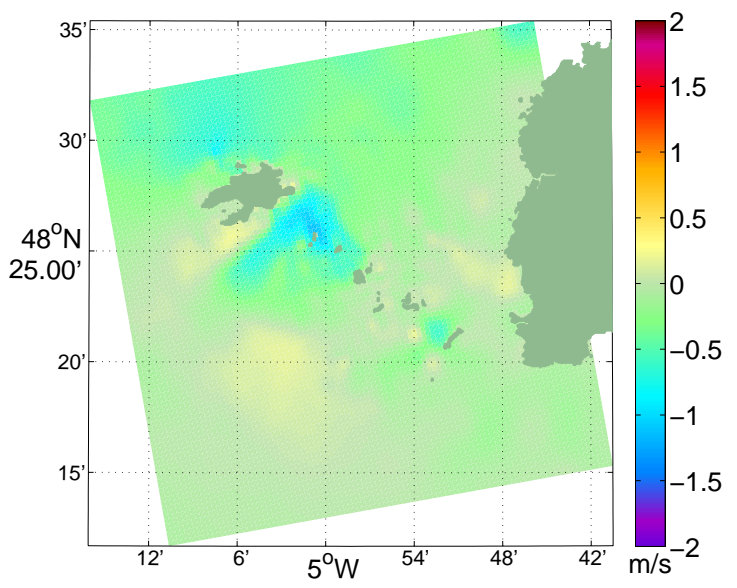

(a)

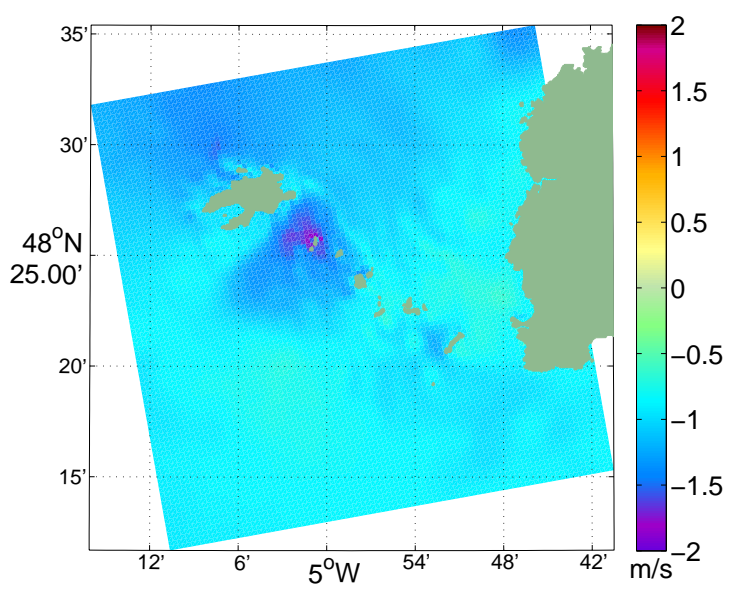

(c)

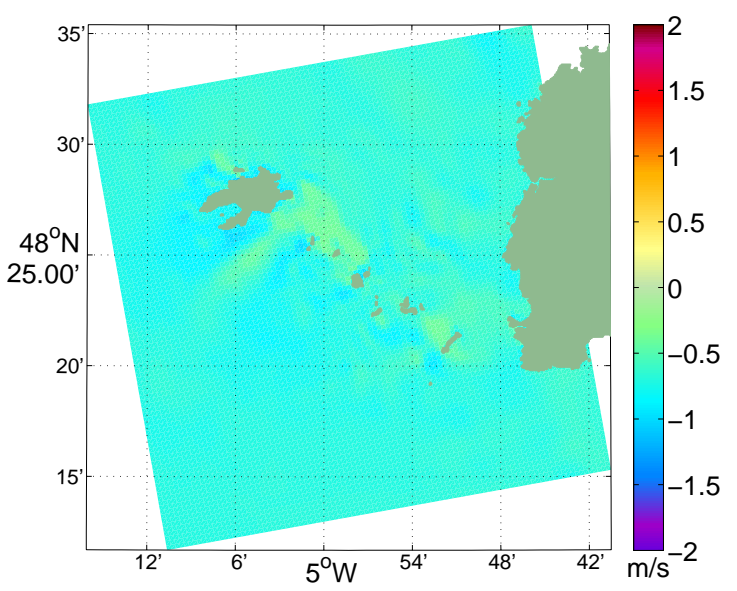

(b)

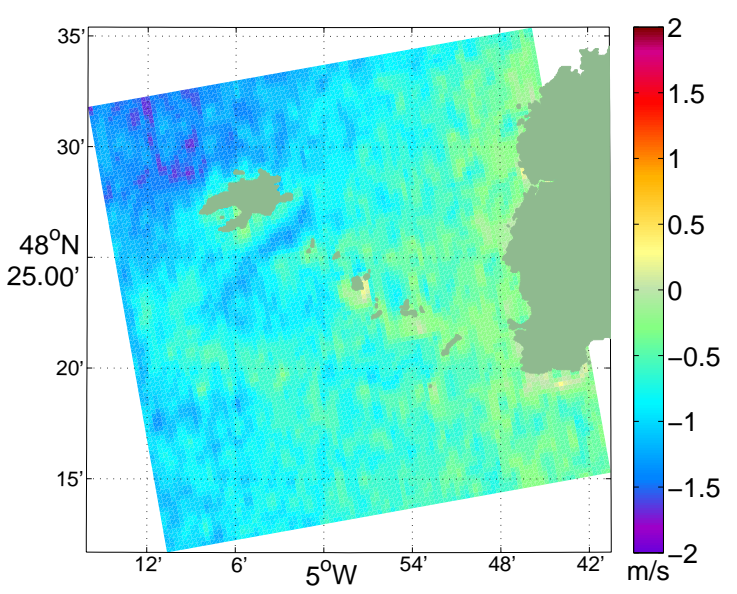

(d)

Figure 6: Projection of model current on SAR look direction (a), contribution from the mean facet velocities and the correlation between wave orbital motion and local NRCS variations (last two terms of (2)) to $V_{D}$ (b), simulated $V_{D}$ (c), and observed $V_{D}$ (d) for the same acquisition as in Fig. 5. Note that the model wind speed in the southwest part of the image is higher than that used for the DopRIM simulations $(4.4 \mathrm{~m} / \mathrm{s})$. This may explain the higher negative signal in the observed range Doppler velocity in the southwest. The accuracy of the observed range Doppler velocity $((\mathrm{d}))$ is about $5 \mathrm{~Hz}$, which corresponds to $22-24 \mathrm{~cm} / \mathrm{s}$ at these incidence angles. 
anomaly patterns are clearly visible in both the simulated and observed range Doppler velocities in the channel between the two islands, with relative speeds ranging from about 1.5 to $2 \mathrm{~m} / \mathrm{s}$. Since the contribution from the surface roughness is significant, strong variability is encountered across the intense current gradient between the islands. This agreement is promising and supports further use of DopRIM simulations in combination with ASAR observations.

\section{Summary and Conclusion}

A radar imaging model (DopRIM) is described and shown to be useful in order to assist in the quantitative investigations of SAR imagery by consistently combining the RIM (Kudryavtsev et al., 2005) with a Doppler shift estimation algorithm. The dependence of the range Doppler velocity on radar parameters and sea state conditions arises via the projected motions of the slightly rough facets, and the line-of-sight velocities of the specular points and breaking crests, as well as the surface current. The strength of this approach lies in the simplified but very efficient separation between the different scattering mechanisms.

Simulated NRCS and range Doppler velocities have been compared to corresponding NRCS and Doppler velocities retrieved from Envisat ASAR WSM data over the Norwegian Sea, as well as an SLC image. Although some discrepancies are revealed, the overall results are encouraging as some inaccuracies in the model current field and near surface wind field are expected. All in all, the results suggest a dominant impact of strong surface currents and their modulation on both the radar-detected surface roughness and the range Doppler signals.

As regular access to range Doppler velocity information and NRCS from ASAR acquisitions over a few selected sites is now possible, the only missing information mostly relates to the limited access to independent surface current measurements for validation. Through such demonstration experiments, DopRIM could be better assessed and explored for transition from a research tool to an operational application in marine monitoring with SAR. Yet, as the range Doppler velocity field, with improved accuracy, is becoming a standard feature of the ground segment on approved and planned SAR missions (such as Sentinel-1), future 
efforts shall be dedicated to assess the potential to better distinguish the different contributions to both radar signal strength and mean Doppler shift. In particular, the differing polarization and/or incidence angle sensitivities can be useful to analyze and filter out the non-Bragg contributions. Also, the combined range Doppler velocity and NRCS with a priori model fields of surface wind, including wind shadowing by land, and current vectors shall offer enhanced possibilities to build better constrained methodologies to more consistently retrieve very high resolution ocean surface information. This will be the topic for future works.

\section{Acknowledgments}

This work was supported by the Nansen Fellowship Foundation at the Nansen Environmental and Remote Sensing Center (NERSC) in Bergen, Norway, by the Research Council of Norway under contract number 177441/V30, by the ESA SAR Wind-Wave and Currents project and the Dragon-2 program under ESRIN contract numbers 18709/05/I-LG and 22494/09/I-LG, and through the ESA Changing Earth Science Network project IncuSAR. An important contribution to the Doppler Radar Imaging Model (DOPRIM) development and simulation work presented in this paper is credited Dr. Dmitry Akimov who sadly passed away on 25 May 2008. Finally we are also grateful to the three anonymous reviewers for their valuable comments and feedback.

\section{References}

Alpers, W., Hasselmann, K., 1978. The Two-Frequency Microwave Technique for Measuring Ocean-Wave Spectra from an Airplane or Satellite. Boundary-Layer Meteorology.

Chapron, B., Collard, F., Ardhuin, F., Jul. 2005. Direct measurements of ocean surface velocity from space: Interpretation and validation. Journal of Geophysical Research Oceans 110 (C9), 7008-+. 
Donelan, M. A., Pierson, W. J., MAY 15 1987. Radar Scattering and Equilibrium Ranges in Wind-Generated Waves with Application to Scatterometry. Journal of Geophysical Research-Oceans 92 (C5), 4971-5029.

Dulov, V., Kudryavtsev, V., Bolshakov, A. N., 2002. A field study of white caps coverage and its modulations by energy containing waves. In: Donelan, M., Drennan, W., Saltzman, E., Wannikhof, R. (Eds.), Gas Transfer at the Water Surface. American Geophysical Union, pp. 187-192.

Hansen, M. W., Collard, F., Dagestad, K.-F., Johannessen, J. A., Fabry, P., Chapron, B., 2011a. Retrieval of Sea Surface Range Velocities from Envisat ASAR Doppler Centroid Measurements. IEEE Transactions on Geoscience and Remote Sensing (in press).

Hansen, M. W., Johannessen, J. A., Dagestad, K.-F., Collard, F.and Chapron, B., 2011 b. Monitoring the Surface Inflow of Atlantic Water to the Norwegian Sea Using Envisat ASAR. Journal of Geophysical Research-Oceans (submitted).

Hughes, B. A., 1978. The Effect of Internal Waves on Surface Wind Waves 2. Theoretical Analysis. Journal of Geophysical Research-Oceans.

Johannessen, J. A., Chapron, B., Collard, F., Kudryavtsev, V., Mouche, A., Akimov, D., Dagestad, K., Nov. 2008. Direct ocean surface velocity measurements from space: Improved quantitative interpretation of Envisat ASAR observations. Geophysical Research Letters 35, 22608-+.

Johannessen, J. A., Kudryavtsev, V., Akimov, D., Eldevik, T., Winther, N., Chapron, B., JUL 22 2005. On radar imaging of current features: 2. mesoscale eddy and current front detection. Journal of Geophysical Research - Oceans 110 (C7).

Kudryavtsev, V., Johannessen, J. A., 2004. On effect of wave breaking on short wind waves. Geophysical Research Letters 31. 
Kudryavtsev, V., Makin, V. K., Klein Tank, A. M. G., Verkaik, J. W., 2000. A model of wind transformation over water-land surfaces. Tech. rep., Royal Netherlands Meteorological Institute (KNMI).

Kudryavtsev, V. N., Akimov, D., Johannessen, J. A., Chapron, B., JUL 22 2005. On radar imaging of current features: 1. Model and comparison with observations. Journal of Geophysical Research-Oceans 110 (C7).

Kudryavtsev, V. N., Hauser, D., Caudal, G., Chapron, B., Jan. 2003a. A semiempirical model of the normalized radar cross-section of the sea surface 1. Background model. Journal of Geophysical Research (Oceans) 108, 8054-+.

Kudryavtsev, V. N., Hauser, D., Caudal, G., Chapron, B., Jan. 2003b. A semiempirical model of the normalized radar cross section of the sea surface, 2. Radar modulation transfer function. Journal of Geophysical Research (Oceans) 108, 8055-+.

Le Nestour, R., 1993. Realisation de l'atlas de courants de mer de la cote ouest de france. Tech. rep., de St-Nazaire a Royan.

Longuet-Higgins, M. S., 1957. The Statistical Analysis of a Random, Moving Surface. Philosophical Transactions of the Royal Society London 613, 321-387.

Lyzenga, D. R., Bennett, J. R., OCT 15 1988. Full-spectrum modeling of synthetic aperture radar internal wave signatures. Journal of Geophysical Research - Oceans 93 (C10), 1234512354.

Madsen, S. N., Mar. 1989. Estimating the Doppler centroid of SAR data. IEEE Transactions on Aerospace Electronic Systems 25, 134-140.

Mouche, A. A., Chapron, B., Reul, N., 2007a. A simplified asymptotic theory for ocean surface electromagnetic wave scattering. Waves in Random and Complex Media 17 (3), $321-341$. 
Mouche, A. A., Chapron, B., Reul, N., Collard, F., 2008. Predicted doppler shifts induced by ocean surface wave displacements using asymptotic electromagnetic wave scattering theories. Waves in Random and Complex Media 18 (1), 185-196.

Mouche, A. A., Chapron, B., Reul, N., Hauser, D., Quilfen, Y., OCT 2 2007b. Importance of the sea surface curvature to interpret the normalized radar cross section. Journal of Geophysical Research-Oceans 112 (C10).

Mouche, A. A., Hauser, D., Kudryavtsev, V., SEP 1 2006. Radar scattering of the ocean surface and sea-roughness properties: A combined analysis from dual-polarizations airborne radar observations and models in C band. Journal of Geophysical Research-Oceans $111(\mathrm{C} 9)$.

Pedersen, F. I., Engen, G., Johnsen, H., 2004. Polarization Dependency in Doppler Frequency Shift and its Application to Envisat ASAR Alt-Pol Data. In: ESA Publication SP-572, Proc. of ERS/Envisat Symposium.

Phillips, O. M., 1980. The Dynamics of the Upper Ocean. Cambridge University Press, Cambridge.

Phillips, O. M., 1984. On the response of short ocean wave components at a fixed wavenumber to ocean current variations. Journal of Physical Oceanography.

Phillips, O. M., 1985. Spectral and Statistical Properties of the Equilibrium Range in WindGenerated Gravity-Waves. Journal of Fluid Mechanics 156 (JUL), 505-531.

Plant, W. J., SEP 15 1986. A 2-Scale Model of Short Wind-Generated Waves and Scatterometry. Journal of Geophysical Research-Oceans 91 (C9), 735-749.

Romeiser, R., Alpers, W., NOV 15 1997. An improved composite surface model for the radar backscattering cross section of the ocean surface .2. model response to surface roughness variations and the radar imaging of underwater bottom topography. Journal of Geophysical Research - Oceans 102 (C11), 25251-25267. 
Romeiser, R., Schmidt, A., Alpers, W., MAY 15 1994. A 3-Scale Composite Surface Model for the Ocean Wave Radar Modulation Transfer-Function. Journal of Geophysical ResearchOceans 99 (C5), 9785-9801.

Romeiser, R., Thompson, D. R., JAN 2000. Numerical study on the along-track interferometric radar imaging mechanism of oceanic surface currents. IEEE Transactions on Geoscience and Remote Sensing 38 (1, Part 2), 446-458.

Rouault, M. J., Mouche, A., Collard, F., Johannessen, J. A., Chapron, B., OCT 2010. Mapping the Agulhas Current from Space: an assessment of ASAR surface current velocities. Journal of Geophysical Research 115 (C10026).

Thompson, D. R., OCT 15 1988. Calculation of Radar Backscatter Modulations From Internal Waves. Journal of Geophysical Research-Oceans 93 (C10), 12371-12380.

\section{Abbreviations and Acronyms}

ASAR Advanced SAR

DopRIM Doppler Radar Imaging Model

HH Horizontal transmit-Horizontal receive

MSS Mean Square Slope

MTF Modulation Transfer Function

NRCS Normalized Radar Cross Section

rhs right-hand-side

RIM Radar Imaging Model

SAR Synthetic Aperture Radar

SLC Single Look Complex 
Vertical transmit-Vertical receive

${ }_{545}$ WSM

Wide Swath Medium resolution image 